\title{
THE SPHERICAL BUILDING AND REGULAR SEMISIMPLE ELEMENTS
}

\author{
G.I. LEHRER
}

\begin{abstract}
Let $G$ be a connected reductive algebraic group defined over a finite field $k$. The finite group $G(k)$ of $k$-rational points of $G$ acts on the spherical building $B(G)$, a polyhedron which is functorially associated with $G$. We identify the subspace of points of $B(G)$ fixed by a regular semisimple element $s$ of $G(k)$ topologically as a subspace of a sphere (apartment) in $B(G)$ which depends on an element of the Weyl group which is determined by $s$. Applications include the derivation of the values of certain characters of $G(k)$ at $s$ by means of Lefschetz theory. The characters considered arise from the action of $G(k)$ on the cohomology of equivariant sheaves over $B(G)$.
\end{abstract}

Let $k$ be the finite field $\mathbb{F}_{q}$ of $q$ elements and $G$ a connected reductive group defined over $k$. In [2] there was constructed a certain topological space $B(G)$ (the construction in [2] applied for an arbitrary field $k$ ) which is associated with $G$ functorially. The (metric) space $B(G)$ is a union of spheres $B(S)$ as $S$ runs over the maximal $k$-split tori of $G$, and has a "rational subspace" which may be roughly thought of as the space of one parameter subgroups of $G$, suitably topologized. In [2] the construction was applied to the derivation of a character formula for the group $G(k)$ of rational points $G(k)$ acting on the homology of $B(G)$; this formula follows from the identification of the fixed-point set

Received 25 January 1983. 
of $g \in G(k)$ on $B(G)$, via Lefschetz theory.

In the present work we prove a general result concerning the fixed point set $B(G)^{s}$ of a regular semisimple element $s \in G(k)$ acting on $B(G)$. This relates $B(G)^{s}$ to the set of points of an, "apartment" $B(S)$ ( $S$ a maximal split torus) which are fixed by an element $w_{s}$ of the Weyl group of $G$ which corresponds to $s$ (see $\$ 2$ below). As an application, we use Lefschetz theory applied to certain subspaces $B_{J}(G)$ ( $J$ a subset of the set $\Pi$ of simple roots) of $B(G)$ to deduce the values of certain principal series characters of $G(k)$ at $s$. One consequence of this is that in the split case, we have $1_{P}^{G(k)}(k)(s)=1_{W_{J}}^{W}\left(w_{s}\right)(J \subset \pi)$. This result is of course not new. Lusztig has proved vastly more general results ([8], [9], [10]) giving the values of all irreducible unipotent characters of $G$ on regular semisimple elements for most groups $G$ and the result has been dealt with explicitly (by different methods) by Deligne and Lusztig [4, $\S 7,8]$, Kawanaka [7] and Surowski [12]. However it seems useful to put it into the present geometric context, at least partly because similar results can be obtained for any representation of $G(k)$ which is realized on the cohomology of an equivariant sheaf over $B(G)$ (see $\$ 6$ below).

Section 1 is devoted to the recollection of the main properties of $B(G)$ and Section 2 to the statement and proof of the main theorem. In Section 3 we introduce certain closed subspaces $B_{J}(G)$ of $B(G)$ and give them a simplicial structure. In Section 4 the homology of the spaces $B_{J}(G)$ is computed simplicially, and in Section 5 the characters of $G(k)$ on the homology groups is studied via the fixed point theory of Section 2 . Finally, in Section 6 , we discuss some special cases, and give a general formulation for $G(k)$-equivariant sheaves over $B(G)$.

\section{The spherical building $B(G)$ of a reductive $k$-group}

Let $G$ be a reductive $k$-group ( $k$ any field). For any maximal $k$-split torus $S$ of $G, B(S)$ is defined as the sphere whose points are the rays (half-lines) of $Y(S) \otimes \mathbb{R}\left(Y(S)=\operatorname{Hom}\left(G_{m}, S\right), G_{m}\right.$ being the 
multiplicative group of $\bar{k})$. To every point $b$ of $B(S)$ we associate a parabolic k-subgroup $P(b)=P_{G}(b)$ of $G$ as in $[2, \S 1]: P(b)$ is the unique parabolic $k$-subgroup of $G$ which contains $S$ and which corresponds to the region of $Y(S) \otimes \mathbf{R}$ in which $b$ lies (these regions are the simplexes of the coxeter complex in the semisimple case).

If $B_{1}$ is the disjoint union of the spheres $B(S)$ (over all maximal $k$-split tori $S$ of $G$ ) then we define an equivalence relation on $B_{1}$ by $b \sim c$ if $c=a d g . b$ for some $g \in P(b)(k)$. The building $B(G)$ is then defined as

(1.1) $B(G)=B_{1}(G) / \sim$.

Clearly $G(k)$ acts on $B(G)$. Some of its principal properties are as follows (these may be found in [2]). Let $S$ be a maximal $k$-split torus of $G$.

(1.2) The projection $B_{1}(G) \rightarrow B(G)=B_{1}(G) / \sim$ restricts to an injection of $B(S)$ into $B(G)$.

(1.3) The point $b(\epsilon B(G))$ is in $B(S)$ if and only if $S \subset P(b)$.

(1.4) The isotropy group of $b$ in $G(k)$ is $P(b)(k)$.

(1.5) Let $s$ be a semisimple element of $G(k)$. Then $B\left(Z_{G}(s)^{0}\right)$ is the fixed point set $B(G)^{s}$ of $s$ acting on $B(G)$.

Suppose now that $k$ is a finite field (say $k=\mathbb{F}_{q}$ as in the introduction). Then ( $c f$. Lusztig [10]) associated with the k-structure of $G$, we have a Frobenius endomorphism $F: G \rightarrow G$ which satisfies

(1.6) $G(k)=G^{F}=\{g \in G \mid F(g)=g\}$;

(1.7) an algebraic subset $H \leq G$ is defined over $k$ if and only if $F(H)=H$;

(1.8) for any $k$-subgroup $H$ of $G$ we have $H(k)=H^{F}$.

\section{Fixed points of regular semisimple elements}

Let $s$ be a regular semisimple element of $G(k)$. Then $s$ lies in a 
unique maximal torus $T$, which is defined over $k$ since $T=Z_{G}(s)$ (cf. $[1, \S \S 10.3,10.5])$.

(2.1) LEMMA. Let $T$ be the maximal toms of $G$ which contains the regular semisimple element $s \in G(k)$. The fixed-point set $B(G)^{s}$ of $s$ acting on $B(G)$ is $B\left(T_{d}\right)$ where $T_{d}$ is the maximal k-split subtorus of $T$.

Proof. From (1.5) we have that $B(G)^{s}=B\left(Z_{G}(s)^{0}\right)$. But $Z_{G}(s)^{0}=T$, whence $B(G)^{s}=B(T)$. From $[2$, Lemma $(7.1)(i)]$ and $[1$, $\$ 1.4$ ] we have that $B(T)=B\left(T_{d}\right)$, whence the result.

The above result applies when $k$ is any field. Henceforth, we take $k=\mathbb{F}_{q}$.

(2.2) LEMMA. A k-torus $R$ of $G$ is $k$-split if and only if $\vec{F}$ acts on $R$ by taking elements to their qth power.

Proof. $R$ is split precisely when there is a $k$-isomorphism $\phi: R \rightarrow D$ where $D$ is a group of diagonal matrices. The Frobenius map on $D$ is given by taking $q$ th powers. Since $\phi$ is a $k$-morphism, it commutes with Frobenius ("transports the $k$-structure"), whence $F$ is also the q-power map on $R$. Conversely, if $F$ consists of taking $q$ th powers, $F$ acts on $Y(R)$ as multiplication by $q$. Hence $Y(R)=Y(R)_{k}$, whence $R$ is split $[1, \S 1.3]$.

(2.2.1) COROLLARY. For any $k$-torus $R$ of $G$, the split part $R_{d}$ of $R$ is given by $R_{d}=\left\{r \in R \mid F(r)=r^{q}\right\}$.

We now fix the following notation:

$S$ a maximal $k$-split torus of $G$;

$\bar{S}$ a maximal $k$-torus of $G$ containing $S$ (so $(\bar{S})_{d}=S$ );

$W=N_{G}(\bar{S}) / \bar{S} \quad$ (the Weyl group of $G$ with respect to $\bar{S}$ );

$k^{W}=N_{G}(S) / Z_{G}(S)$ 
$\dot{w}$ is a representative in $N_{G}(\bar{S})$ for $w \in W$

We give a proof of the following result of Springer and Steinberg [13, II.1.2] for the reader's convenience as well as for the purpose of establishing notation.

(2.3) LEMMA. The $G(k)=G^{F}$-conjugacy classes of F-stable maximal tori of $G$ are in bijective correspondence with the F-classes of $W$, where F-equivalence is defined by $w_{1} \sim_{F} w_{2}$ if $w_{2}=v w_{1} F(v)^{-1}$, some $v \in W$.

Proof. Let $S_{1}$ be any $F$-stable maximal torus in $G$. Then $S_{1}=g \bar{S} g^{-1}$, some $g \in G ;$ since $S_{1}$ and $\bar{S}$ are $F$-stable, we have $F(g) \bar{S} F(g)^{-1}=g^{S} g^{-1}$ whence $g^{-1} F(g)=\dot{w} \in N(\bar{S})$. It is easily checked that replacing $g$ by $g n(g \in N(\bar{S}))$ or $S_{1}$ by a $G$-conjugate has the effect of replacing $w$ by an F-equivalent element of $W$.

Now suppose that $s$ is a regular semisimple element of $G(k)$ and that $T$ is the unique maximal torus of $G$ such that $s \in T$. Then $T$ is $F$-stable (see above) and so by (2.3), $T$ corresponds to an element $w \in W$, which is determined to within $F$-conjugacy. Replacing $s$ by a $G(k)$-conjugate clearly gives the same $F$-class. We have shown:

(2.4) PROPOSITION. To each $G(k)$-conjugacy class of regular semisimple elements of $G(k)$ there corresponds a wique F-conjugacy class in $W$.

(2.5) THEOREM. Let $s$ be a regular semisimple element of $G(k)$ and let $c_{s}$ be the corresponding (cf. (2.4)) F-class in $W$. Then the fixedpoint set $B(G)^{s}$ is $G(k)$-conjugate to $B\left(Z_{S}\left(w_{s}\right)\right)$ for some element $w_{s} \in c_{s}$, where $z_{S}\left(w_{s}\right)=\left\{t \in S \mid \dot{w}_{s}=\dot{w}_{s} t\right\}$ and $s$ is the maximal $k$-split torus of $G$ fixed above.

Proof. If $T$ is the unique maximal torus of $G$ which contains $b$, and $T=g \bar{S} g^{-1}$, then $g^{-1} F(g)=\dot{w}$ and $w \in c_{s}$ (see (2.3)). 
Now $B(G)^{S}=B\left(T_{d}\right)$ by $(2.1)$, and since $T_{d}$ is $k$-split, there is an element $x$ of $G(k)$ such that ${ }^{x_{T}} \subset S$ (all maximal $k$-split tori are $G(k)$-conjugate by $[1, \S 4.21])$. But $B(G)^{x s x^{-1}}=a d x . B(G)^{s}$ for any $x \in G(k)$, whence replacing $s$ (and therefore $T$ ) by a $G(k)$-conjugate replaces $B(G)^{S}$ by a $G(k)$-conjugate and fixes the $F$-class $c_{s}$. Thus we may assume that $T_{d} \subset S$.

After this reduction, we have that $\bar{S}$ and $T$ are both maximal $k$-tori in $Z_{G}\left(T_{d}\right)$, which is a connected reductive $k$-subgroup of $G[1$, (2.15) (d)]. Thus there is an element $z \in Z_{G}\left(T_{d}\right)$ such that $T=z \bar{S} z^{-1}$ and the element $w_{s} \in W$ defined by $\dot{w}_{s}=z^{-1} F(z)$ is in the $F$-class $c_{s}$ of $W$.

Moreover since $z$ centralizes $T_{d}$, so does $F(z)$ and hence so does $\dot{w}_{s}=z^{-1} F(z)$. Thus $T_{d} \leq Z_{S}\left(w_{s}\right)$. Conversely, suppose that $x \in S$ is fixed by $w_{s}$. Then $z^{-1} F(z) x F(z)^{-1} z=x$, whence $z x z^{-1}=F(z) x F(z)^{-1}$. Taking qth powers of both sides, we see that

$$
\left(z x z^{-1}\right)^{q}=F(z) x^{q} F(z)^{-1}=F\left(z x z^{-1}\right)
$$

since by $(2.2)$ we have that $x^{q}=F(x)$.

But $z x z^{-1} \in z S z^{-1} \subset z \bar{S} z^{-1}=T$. It follows from (2.2.1) (since $\left.F\left(z x z^{-1}\right)=\left(z x z^{-1}\right)^{q}\right)$, that $z x z^{-1}$ is in the split part of $T$, that is $z x z^{-1} \in T_{d}$. Since $z$ centralizes $T_{d}$, it follows that $x \in T_{d}$ and so $z_{S}\left(w_{s}\right) \leq T_{d}$. Hence $z_{S}\left(w_{s}\right)=T_{d}$ and the theorem is proved.

(2.6) COROLLARY. Suppose $G$ is $k$-split and that $s$ is a regular semisimple semisimple element of $G(k)$. Then $B(G)^{s}$ is $G(k)$-conjugate to $B(S)^{w_{s}}$, for any element $w_{s} \in c_{s}$, where $c_{s}$ is the conjugacy class of $W$ corresponding to $s$. 
Proof. In this case $S=\bar{S}$ and $F$ acts trivially on $W$. Thus the $F$-conjugacy classes of $W$ are simply the conjugacy classes and the statement follows directly from (2.5).

We conclude this section with the following characterization of $B\left(z_{S}(w)\right)$, for $w \in W$.

(2.7) PROPOSITION. Let $w \in W$. A point $b$ of $B(S)$ is in $B\left(z_{S}(w)\right)$ if and only if $\dot{w} \in P(b)$.

Proof. Write $S^{\prime}=Z_{S}(w)$. This is a $k$-split torus of $G[1, \S 1.6]$ and from [2, Lemma (1.2) (ii)] we have that $b \in B\left(S^{\prime}\right)$ implies that $P(b) \supset Z_{G}\left(S^{\prime}\right)$, so that $\dot{w} \in P(b)$.

Conversely, suppose that $b \in \mathrm{B}(S)$ and $\dot{w} \in P(b)$. Then $S \cap \operatorname{Rad} P(b)$ centralizes $\dot{w}$ and from [2, Lemma (1.2) (ii)] we have $b \in B(S \cap \operatorname{Rad} P(b))$. Since $S \cap \operatorname{Rad} P(b) \subset Z_{G}(\dot{w})$, we have $b \in B\left(S \cap Z_{G}(\dot{w})\right)=B\left(Z_{S}(\dot{w})\right)$ as required.

\section{The subspaces $B_{J}$}

Let $k$ be an algebraic closure of $k$; we write $\Phi, \Phi^{+}$, $\Pi$ for the set of roots, positive roots and simple roots of $G$ determined by $\bar{S}$ and a Borel subgroup $B \supset \bar{S}$ ( $B$ may be assumed to be a $k$-group). The corresponding Weyl group is $W=W(\bar{S}, G)$. Following Borel and Tits [2, $\S 5]$, the corresponding data for the $k$-structure will be denoted $k^{\Phi}=\Phi(S, G), k^{\Phi^{+}}, k^{\Pi}$ and $k^{W}$.

The following facts will be of importance later.

(3.1) The parabolic $k$-subgroups of $G$ which contain $B$ are the ${ }_{k}^{P} J$ for the various subsets $J \subset{ }_{k} \Pi[1, \S 5.12]$.

(3.2) The parabolic $k$-subgroups of $G$ which contain $S$ are

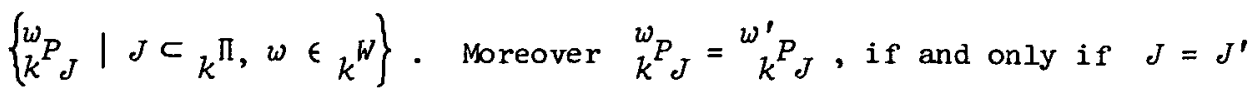
and $w_{W_{J}}={ }^{w^{\prime}} W_{J}$ (here ${ }_{k}^{w_{P}}=w\left({ }_{k} P_{J}\right) w^{-1}$, and so on).

This follows from $[1, \S \S 5.9$ and 5.15$]$. 
(3.3) For any subset $J \subset{ }_{k} \Pi$, there is a unique $F$-invariant subset $\bar{J} \subset I I$ such that $k^{P}{ }_{J}=P_{\bar{J}}$.

Since $k_{J} P_{J}$ contains $B$, clearly $k_{J} P_{J}=P_{J}$ for some $\bar{J} \subset \Delta$. Moreover, since $k^{P} J$ is defined over $k$, it is $E$-invariant, whence it follows that $W_{J}$ is $F$-invariant. But $F$ maps $I$ to itself, whence $E(\bar{J})=\bar{J} \quad[13$, II. 14$]$.

For each subset $J \subset \subset_{k} \Pi$ we define a $G(k)$-invariant subspace $B_{J}(G)$ as follows.

(3.4) DEFINITION.

(i) $B_{J}(G)=\left\{b \in B(G) \mid P(b) \geq g\left(k_{k} P_{J}\right) g^{-1}\right.$ for some $\left.g \in G(k)\right\}$.

(ii) For any reductive $k$-subgroup $H \leq G$, define $B_{J}(H)=B(H) \cap B_{J}(G)$.

Theorem (2.5) may be restated for $B_{J}$ as follows.

(3.5) PROPOSITION. Let $s$ be a regular semisimple element in $G(k)$, with corresponding $F$-conjugacy class $c_{s}$ in $W$. There exists $w_{s} \in c_{s}$ such that

$$
B_{J}(G)^{s}=B_{J}(G) \cap B\left(z_{S}\left(w_{S}\right)\right)
$$

To investigate the topological nature of $B_{J}(G)$ and $B_{J}(S)$ we introduce the following finite simplicial complexes.

$\Delta_{J}=\Delta_{J}(G, k)$ is the subcomplex of the combinatorial building (see $[2, \S 6])$ consisting of the following simplexes:

(3.6) $\Delta_{J}=\left\{P \mid P\right.$ is a parabolic $k$-subgroup of $G, P \supset{ }_{k}^{g} P_{J}$ for some $g \in G(k)\}$.

Analogously, we introduce the subcomplex $\Gamma_{J}$ of the Coxeter complex $\Gamma$ of $k^{W}$ as follows. 
(3.7) $\Gamma_{J}=\left\{{ }_{k} W^{w} \mid L \supset J, w \epsilon_{k} W\right\}$.

(3.8) PROPOSITION. If $G$ is semisimple, then there is a $G(k)$ equivariant homeomorphism $\tau:\left|\Delta_{J}(G)\right| \rightarrow B_{J}(G)$ satisfying

(i) for $b \in\left|\Delta_{J}(G)\right|, P(\tau(b))$ is the parabolic subgroup corresponding to the simplex of $\Delta_{J}$ containing $b$ in its interior;

(ii) if $S$ is a maximal k-split torus and $\Delta_{J}(S)$ is the subcomplex of. $\Delta_{J}(G)$ whose simplexes correspond to the parabolic k-subgroups containing $S$, then $\tau$ restricted to $\left|\Delta_{J}(S)\right|$ is a triangulation of $B_{J}(S)$.

The proof consists of the observation that the map $\tau$ of $[2$, Proposition (6.1)] takes $\left|\Delta_{J}\right|$ to $B_{J}$ and $\left|\Delta_{J}(S)\right|$ to $B_{J}(S)$.

In addition we have

(3.9) PROPOSITION. Let $G^{\prime}$ be the derived group of $G$, and let $d$ be the $k$-rank of the connected centre of $G$. Then $B_{J}(G)$ may be identified in a $G(k)$-equivariant way with the $d$-fold suspension of $B_{J}\left(G^{\prime}\right)$. This identification maps $B_{J}(S)$ (S a maximal k-split torus of $G)$ to the d-fold suspension of $B_{J}\left(S^{\prime}\right)$, where $S^{\prime}$ is a maximal $k$-split torus of $G^{\prime}$, which is contained in $S\left(S^{\prime}=S \cap G^{\prime}\right)$.

The proof is an immediate consequence of the proof of (7.1) and (7.2) in [2].

Combining (3.9) and (3.8), we obtain

(3.10) COROLLARY. (i) $B_{J}(G)$ is homeomorphic in a $G(k)$-equivariant way with the d-fold suspension of $\left|\Delta_{J}\right|$.

(ii) $B_{J}(S)$ is homeomorphic in a $k^{W-e q u i v a r i a n t ~ w a y ~ t o ~ t h e ~} d$-fold suspension of $\left|\Gamma_{J}\right|$. 
4. The simplicial complexes $\Gamma_{J}$ and $\Delta_{J}$

Although similar results are fairly well known (see, for example, [16]), we include the proof of the following result for completeness. The proof follows ideas of Solomon [12] who treated the case $J=\emptyset$.

(4.1) PROPOSITION. Suppose $\left|{ }_{k} \Pi-J\right|>I$. Then the complex $\Gamma_{J}$ has rational homology given by

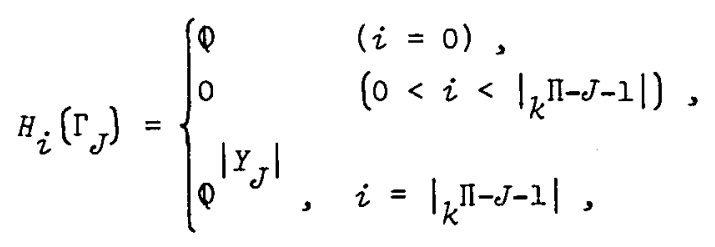

where $y_{J}=\left\{w \in \epsilon_{k} \mid w^{-1}(J) \subset_{k} \Phi^{+}, w^{-1}\left({ }_{k} \Pi-J\right) \subset_{k} \Phi^{-}\right\}$.

We use the following elementary result, which may be found in Solomon [12].

(4.1.1) LEMMA. Let $K$ be a simplicial complex and $L, L_{1}, \ldots, L_{n}$ be subcomplexes such that

(i) $K=L \cup L_{1} \cup \ldots \cup L_{n}$,

(ii) $L_{i}$ has the homology of a point (each $i$ ),

(iii) $L \cap L_{i}$ has the homology of a point (each $i$ ),

(iv) $L_{i} \cap L_{j} \subset L$ if $i \neq j$.

Then $H_{*}(K) \cong H_{*}(L)$.

In the proof of (4.1) we shall use the following notation: for any simplex $\sigma$, we denote by $[\sigma]$ the complex consisting of $\sigma$ together with its faces; a chamber of a simplicial complex $K$ is a simplex of maximal dimension; for any subset $L \subset{ }_{k} \Pi, X_{L}$ denotes the set of shortest right coset representatives of $k^{W}$ in $k^{W}$, that is, $x_{L}=\left\{w \in W \mid w^{-1}(L) \subset{ }_{k} \Phi^{+}\right\}$. Clearly $x_{J}$ is a disjoint union: $X_{J}=\underset{L}{\dot{U}} Y_{L}$ 
For any simplex $\sigma \in \Gamma$, define the distance $d(\sigma)$ of $\sigma$ from the fundamental chamber [1] by $d(\sigma)=\imath(\omega)$, where $w$ is the shortest element in $\sigma$. Write $\Gamma_{h}=\{\sigma \in \Gamma \mid d(\sigma) \leq h\} \quad$ ( $h$ a positive integer $)$, and correspondingly, write $\Gamma_{J, h}=\Gamma_{J} \cap \Gamma_{h}$.

(4.1.2) LEMMA. Let $\sigma={ }_{k}{ }^{W} L^{w}$ be a simplex of $\Gamma, w \in X_{L}$. Then

(i) the faces of $\sigma$ of codimension 1 are

$$
\left\{_{k} W_{L \cup\{r}\right\}^{w} \mid r=r_{\alpha}, \alpha \in k^{\Pi-L\}}:
$$

for any face $\sigma^{\prime}$ of $\sigma$, we have $d\left(\sigma^{\prime}\right) \leq d(\sigma)$;

(ii) the face $k^{W^{\prime}}, w=\sigma^{\prime}$ of $\left({ }_{k} \Pi \supset L^{\prime} \supset L\right)$ satisfies $d\left(\sigma^{\prime}\right)<d(\sigma)$ if and only if there is an element $\alpha \in L^{\prime}-L$ such that $Z\left(r_{\alpha} w\right)<Z(w)$;

(iii) if $d(\sigma)>0$, then $\sigma$ has a face $\sigma^{\prime}$ of codimension 1 with $d\left(\sigma^{\prime}\right)<d(\sigma)$.

The proof of (4.1.2) is an easy exercise in Weyl groups. As an immediate consequence, we see that $\Gamma_{h}$ and $\Gamma_{J, h}$ are subcomplexes of $\Gamma$ and $\Gamma_{J}$ respectively.

(4.1.3) COROLLARY. Let $\sigma=k^{W} L^{w}$ be a simplex of $\Gamma$, and assume $w \in X_{L}$. Then $\sigma$ has a proper face $\sigma^{\prime}$ with $d\left(\sigma^{\prime}\right)=d(\sigma)$ if and only if $w \notin Y_{L}$.

Proof. If $w \notin Y_{L}$ then there is an element $\alpha \epsilon_{k}$ I-L such that if $r=r_{\alpha}$ is the corresponding reflection in $k^{W}$, then $Z(r w)>Z(w)$. By (4.1.2) (ii), the face $\sigma_{r}=k^{W} L \cup\{r\}^{w}$ then satisfies $d\left(\sigma_{r}\right)=d(\sigma)$.

The converse follows similarly.

(4.1.4) LEMMA. With notation as in (4.1.3), suppose that $d(\sigma)=h$. Then

$$
\text { [б] } \cap \Gamma_{h-1}=\bigcup_{i=1}^{p}\left[\sigma_{i}\right]
$$


where $\sigma_{1}, \ldots, \sigma_{p}$ are the faces of $\sigma$ which have codimension 1 and satisfy $d\left(\sigma_{i}\right)<h$.

This follows easily from (4.1.2) (ii).

Let $C\left(\Gamma_{J}\right)$ be the collection of simplexes of $\Gamma_{J}$ of maximal dimension $\left(=\left\{_{k} W_{J} v \mid v \in_{k} W\right\}\right)$. Write $C^{0}\left(\Gamma_{J}\right)$ for the set of simplexes $\sigma$ in $C\left(\Gamma_{J}\right)$ which have a (proper) face $\sigma^{\prime}$ such that $d\left(\sigma^{\prime}\right)=d(\sigma)$. By (4.1.3), we have that $C^{0}\left(\Gamma_{J}\right)=\left\{k_{J} J^{v} \mid v \in X_{J}-Y J\right\}$. Correspondingly, we write $\Gamma_{J}^{0}=\bigcup_{\sigma \in C^{0}\left(\Gamma_{J}\right)}^{U}[\sigma]$.

(4.1.5) LEMMA. Suppose $\left|{ }_{k}^{\Pi-J}\right|>1$. Then $\Gamma_{J}^{0}$ has the homology of a point.

Proof. Write $\Gamma_{J, h}^{0}=\Gamma_{J}^{0} \cap \Gamma_{h}(h=0,1,2, \ldots) \cdot$ Clearly $\Gamma_{J, 0}^{0}=\left[W_{J}\right]$ is contractible. We show by induction on $h$ that $\Gamma_{J, h}^{0}$ has the homology of a point for all $h$. Now

$$
\Gamma_{J, h+1}=\Gamma_{J, h}^{0} \cup\left[{ }_{k}{ }^{W} w_{1}\right] \cup \ldots \cup\left[{ }_{k}{ }^{2} w_{m}\right]
$$

where $\left\{k_{k} w_{i} \mid i=1, \ldots, m\right\}=\left\{\sigma \in c^{0}\left(\Gamma_{J}\right) \mid d(\sigma)=h+1\right\}$. One now checks the conditions of Lemma (4.1.1): (i) and (ii) are trivial; thus it remains to verify

(iii) $\left[\sigma_{i}\right] \cap \Gamma_{J, h}^{0}$ has the homology of a point $\left(\sigma_{i}=k^{W} w_{i}\right)$ and

$$
\text { (iv) }\left[\sigma_{i}\right] \cap\left[\sigma_{j}\right] \subset \Gamma_{J, h}^{0} \text { if } i \neq j \text {. }
$$

For (iii), we have from (4.1.4) that $\left[\sigma_{i}\right] \cap \Gamma_{J, h}^{0}=\bigcup_{j=1}^{p}\left[\tau_{i j}\right]$ where $\tau_{i_{1}}, \ldots, \tau_{i_{p}}$ are the faces of $\sigma_{i}$ which have codimension 1 and satisfy $d\left(\tau_{i j}\right) \leq h$. Now by $(4.1 .2)(i i i), \sigma_{i}$ has a face $\tau_{i l}$ with 
$d\left(\tau_{i l}\right) \leq h$. Further, since $\sigma_{i} \in \Gamma_{J}^{0}, \sigma_{i}$ also has a face $\tau$ with $d(\tau)=h+1$. Thus $\bigcap_{j=1}^{p}\left[\tau_{i j}\right] \neq \emptyset\left(\partial_{k}{ }^{W} \Pi_{k}-\{r\}^{w}\right.$, where $\tau=k^{W} J u\{r\}^{\omega}{ }_{i}$ is any face satisfying $\left.d(\tau)=d\left(\sigma_{i}\right)\right)$, and it follows that $\bigcup_{j=1}^{p}\left[\tau_{i j}\right]$ is contractible. Condition ( $i v)$ is easily verified. Thus Lemma (4.1.1) applies, and we deduce that $\Gamma_{J, h}^{0}$ and $\Gamma_{J, h+l}^{0}$ have the same homology. The result follows.

(4.1.6) LEMMA. The complexes $\Gamma_{J}$ and $\Gamma_{J}^{0}$ have the same $(d-1)-$ skeleton, where $d=\operatorname{dim} \Gamma_{J}=\left|k_{k} \Pi-J\right|-1$.

Proof. Let $k^{W} J \cup\{r\}^{w}$ be a (d-I)-simplex of $\Gamma_{J}$; we may assume $w$ to be in $X_{J \cup\{\alpha\}}$, where $r=r_{\alpha}$. Then a fortiori, $w \in X_{J}$ and it follows that the simplex $\sigma={ }_{k} W J$ lies in $C^{0}\left(\Gamma_{J}\right)$ (it has the face $\sigma^{\prime}=k^{W} J \cup\{r\}^{w}$ with $\left.d\left(\sigma^{\prime}\right)=d(\sigma)\right]$. Thus $\sigma^{\prime} \in[\sigma] \subset \Gamma_{J}^{0}$.

Proof of (4.1). From (4.1.6), we have

$$
\Gamma_{J}=\Gamma_{J}^{0} \cup\left\{\omega_{1}\right\} \cup\left\{\omega_{2}\right\} \cup \ldots \cup\left\{\omega_{t}\right\}
$$

where $\omega_{i}=k^{W} y_{i}$ and $y_{J}=\left\{y_{1}, \ldots, y_{t}\right\}$. Thus

$$
H_{J}\left(\Gamma_{J}\right)= \begin{cases}H_{p}\left(\Gamma_{J}^{0}\right) & (p<d), \\ H_{p}\left(\Gamma_{J}^{0}\right) \oplus 0^{\left|Y_{J}\right|} & (p=d) .\end{cases}
$$

The result now follows immediately from (4.1.5).

From (4.1) we quickly deduce the homology of $\Delta_{J}$ using

(4.2) LEMMA. The partially ordered sets $k^{W \backslash\left(\Gamma_{J} \times \Gamma_{J}\right)}$ and $G(k) \backslash\left(\Delta_{J} \times \Delta_{J}\right)$ are isomorphic.

Proof. The proof is exactly the same as that of the corresponding 
result for $k^{W \backslash(\Gamma \times \Gamma)}$ and $G(k) \backslash(\Delta \times \Delta)$. It may be found in [3].

(4.3) COROLLARY. Suppose ||$_{k} \Pi-J \mid>1$. Then then homology of $\Delta_{J}$ is given by

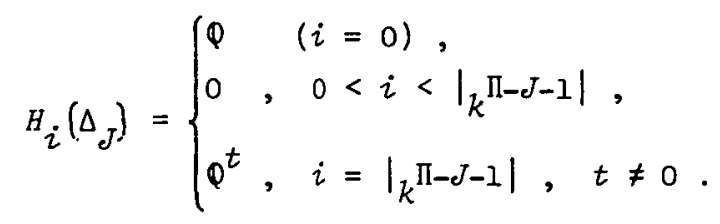

Putting together the results of (3.11), (4.1) and (4.3) we obtain (4.4) THEOREM. Let $J \subset{ }_{k} \Pi$, with $D=||_{k} \Pi-J \mid+d-1>0$ where $d=k$-rank of $Z(G)$. Then the rational homology groups of $B_{J}(G)$ and $\mathrm{B}_{J}(S)$ are given by

$$
\begin{aligned}
& H_{i}\left(B_{J}(G)\right)= \begin{cases}\varphi, & i \neq 0, \\
0, & 0<i<D, \\
Q^{t}, & t \neq 0, \quad i=D,\end{cases} \\
& H_{i}\left(B_{J}(S)\right)= \begin{cases}Q, & i \neq 0, \\
0, & 0<i<D, \\
\left|Y_{J}\right|, & i=D,\end{cases}
\end{aligned}
$$

where $Y_{J}=\left\{w \in k_{k}^{W} \mid w J \subset \Phi^{+}, w\left({ }_{k} \Pi-J\right) \subset \Phi^{-}\right\}$.

\section{Representations associated with the spaces $B_{J}(G)$}

From Theorem (4.4) it is apparent that we have a representation $M_{J}$ of $G(k)$ on $H_{D}\left(B_{J}(G)\right)$. Moreover when $\left|{ }_{k} \Pi-J\right|>1$, it follows from (3.10) (i) that $M_{J}$ is the same representation as that of $G(k)$ on $\left.{ }^{H}\right|_{k} \Pi-J \mid-1\left(\Delta_{J}(G, k)\right)$. Applying the Hopf trace formula to this latter complex we see that (when $|k \Pi-J|>1$ )

$$
\text { (5.1) } M_{J}=\sum_{L, k \amalg L J}(-1)^{|L-J|_{\operatorname{Ind}} G(k)}{ }_{k L}{ }_{L}(k)(1) \text {. }
$$


Moreover, we may always assume that the $k$-rank $d$ of $2(G)^{0}$ is nonzero (by suspending $B(G)$ if necessary); thus the formula (5.1) holds without restriction on $J$. This is equivalent to stipulating that $B_{J}(G)$ is connected for all $J$.

The representations $M_{J}$ were introduced by Solomon [11] and studied by Surowski [16] and Stanley [14]. Our object in reintroducing them here is to show how the trace of $M_{J}$ at regular semisimple elements of $G(k)$ may be evaluated directly from our main result (Theorem (2.5)).

(5.2) DEFINITION. For ' $L \subset{ }_{k} \Pi$ define $\alpha_{L}: W \rightarrow \mathbb{N}$ by

$$
\alpha_{L}(x)=\#\left\{W_{\bar{L}}^{\omega} \mid \omega \epsilon_{k}^{W}, W_{L}^{-\omega x}=W_{\bar{L}}^{\omega}\right\}
$$

Note that in the split case, $\alpha_{L}=\operatorname{Ind}_{W_{L}}^{W}(1) \quad\left(W=k^{W}, L=\bar{L}\right)$.

(5.3) THEOREM. Let $s$ be a regular semisimple element of $G(k)$, and let $w_{s}$ be the corresponding element of $W$, chosen as in the statement of Theorem (2.5). If $\mu_{J}$ is the character of the representation $M_{J}$ above, then

$$
\mu_{J}(s)=\sum_{k \in L \subset J}(-1)^{|L-J|} \alpha_{L}\left(w_{s}\right) .
$$

Proof. We have by the Lefschetz principle that

$$
\sum_{i=0}^{D}(-1)^{i} \operatorname{tr}\left(s, H_{i}\left(B_{J}(G)\right)\right)=\chi_{E}\left(B_{J}(G)\right)^{s},
$$

where $\chi_{E}$ is the Euler characteristic.

But by (4.4) the left side reduces to $1+(-1)^{D} \mu_{J}(s)$. Now by Theorem (2.5), the right hand side is

$$
\mathrm{X}_{E}\left[\mathrm{~B}_{d}(G) \cap B\left(\mathrm{Z}_{S}\left(w_{s}\right)\right)\right]=\mathrm{X}_{E}\left[\mathrm{~B}_{J}\left(\mathrm{Z}_{S}\left(w_{s}\right)\right)\right] \text {. }
$$

To identify the topological nature of the space $B_{J}\left(z_{S}\left(w_{S}\right)\right)$ we wish to relate it to the complex $\Gamma_{J}(c f .(3.10)(i i))$. For this we use (2.7) to 
prove

(5.4) LEMMA. Let $\Gamma_{J}^{w}$ be the subcomplex of $\Gamma_{J}$ defined by the condition

$$
W_{L} w \in \Gamma_{J}^{w} \Leftrightarrow w_{L}^{w w_{S}}=w_{\tau^{\omega}}
$$

Then under the identification of (3.10) (ii), $B_{J}\left(z_{S}\left(w_{s}\right)\right)$ is identified with the d-fold suspension of $\left|\Gamma_{J}^{w}\right|$.

Proof of lemma. From (2.7) we have that $b \in B(S)$ is in $B\left(z_{S}\left(w_{s}\right)\right) \Leftrightarrow \dot{w}_{S} \in P(b)$. Now for $b \in B_{J}(S), P(b)=w^{-1} P_{L} w$ for some $L \in{ }_{k} \Pi$, with $L \supset J\left(\right.$ recall $\left.{ }_{k} P_{L}=P_{L}\right)$, and some $w \epsilon_{k} W$. Thus the condition $\dot{w}_{s} \in P(b)$ is equivalent to $w_{s} \in w^{-1}{ }_{L}^{w}$, or that $W_{L} w \omega_{s}=W_{L}^{w}$. Thus under the identification of (3.10) (ii), $B_{J}\left(z_{S}\left(w_{s}\right)\right)$ is precisely the image of the suspensions of the simplexes in $\Gamma_{J}^{w}$. This proves the lemma.

Completion of the proof of Theorem (5.3). The Euler characteristic of $B_{J}\left(z_{S}\left(w_{s}\right)\right)$ is now easily computed in terms of that of $\Gamma_{J}^{w_{s}}$, which is obtained by using the Hopf trace formula, and recalling that $\chi_{E}(S X)=2-X_{E}(X)$, where $S X$ is the suspension of the topological space $X$.

(5.5) COROLLARY. We have, for each subset $J \subset{ }_{k}^{\Pi}$,

$$
\underset{{ }_{k} P_{J}(k)}{G(k)}(1)(s)=\alpha_{J}\left(w_{s}\right) .
$$

This is obtained by applying Möbius inversion with respect to the partially ordered set of subsets of $k^{\Pi}$ to the formula of (5.3). 


\section{Concluding remarks}

(6.1) It is not immediately apparent that our definition of $\alpha_{L}(x)$ is F-conjugacy invariant. This is because the element $w_{s}$ is somewhat special. However, let us define

$$
\alpha_{L}^{\prime}(x)=\#\left\{W_{\vec{L}} \mid W_{\bar{L}} \omega x=W-F(x)\right\} .
$$

It is then not difficult to show that for the elements $w_{s}$ of (5.5), one has $\alpha_{L}\left(w_{s}\right)=\alpha_{L}^{\prime}\left(w_{s}\right)$, and $\alpha_{L}^{\prime}$ is patently F-conjugacy invariant: $\alpha_{L}^{\prime}\left(\omega x F(w)^{-1}\right)=\alpha_{L}^{\prime}(x)$ for each $w \in W$.

(6.2) Next, we remark that in the split case (when $F$ acts trivially on $W$ and $S=\bar{S}$ in $\S 2$ ) the results (and proofs) simplify. In particular, in the split case, one has a $W$-action on $B(S)$ and Theorem (2.5) has the simple formulation given in (2.6).

The result (5) is then deduced from the simple geometric observation that

(5.5)' we have $\Lambda\left(s, B_{J}(G)\right)=\Lambda\left(w_{s}, B_{J}(S)\right) \quad$ (where $\Lambda(-, X)$

$$
\text { denotes the Lefschetz number: } \left.\sum_{i=0}^{\infty}(-1)^{i} \operatorname{tr}\left(-, H_{i}(X)\right) .\right)
$$

(6.3) As mentioned in the introduction, the formula (5.5) is not new. One of the by-products of the present geometric setting for it is that it may be generalized to the case where we have an equivariant $G(k)$-sheaf $F$ of complex vector spaces on $B(G)$ (in the sense of Grothendieck [6]). Suppose for simplicity that $G$ is split; we assume always that the cohomology modules $H_{c}^{i}(B(G), F)$ (cohomology with compact supports) are of finite type (that is, have finite complex dimension).

One then has a virtual $G(k)$ module $\Lambda_{G}=\sum_{i=0}^{\infty}(-1)^{i} H_{c}^{i}(B(G), F)$ whose associated trace function will be written $\lambda_{G}(B(G), F)(x)$. Now the methods of Verdier [17] may be generalized (see Donovan and Lehrer [5]) to prove that 


$$
\lambda_{G}(B(G), F)(x)=\lambda_{\langle x\rangle}\left(B(G)^{x}, F \mid B(G)^{x}\right)(x),
$$

where $\langle x\rangle$ denotes the cyclic group generated by $x$ and $F \mid Y$ denotes the sheaf $F$ restricted to the subspace $Y$ of $B(G)$. Hence for $s$ a regular semisimple element of $G(k)$ (and $G$ split), we have

$$
\text { (6.4) } \lambda_{G}(B(G), F)(s)=\lambda_{\left(s^{\prime}\right)}\left(B(S)^{w} s, F \mid B(S)^{w} s\right)\left(s^{\prime}\right)
$$

where $s^{\prime}$ is an appropriate $G(k)$-conjugate of $s$.

We note finally that since $B_{J}(G)$ is a closed subspace of $B(G)$, the discussion in $\$ 5$ may be thought of as a special case of $(6.4)$, where $F$ is taken as constant on $B_{J}(G)$ and zero outside $B_{J}(G)$.

\section{References}

[1] Armand Borel et Jacques Tits, "Groups réductifs", Inst. Hautes Études Sci. Publ. Math. 27 (1965), 55-151.

[2] C.W. Curtis, G.I. Lehrer, and J. Tits, "Spherical buildings and the character of the Steinberg representation", Invent. Math. 58 (1980), 201-210.

[3] C.W. Curtis and G.1. Lehrer, "A new proof of a theorem of solmonTits", Proc. Amer. Math. Soc. 85 (1982), 154-156.

[4] P. Deligne and G. Lusztig, "Representations of reductive groups over finite fields", Ann. of Math. (2) 103 (1976), 103-161.

[5] P.W. Donovan and G.I. Lehrer, "On the Euler-Poincaré characteristic of a G-sheaf", in preparation.

[6] Alexandre Grothendieck, "Sur quelques points d'algèbe homologique", Tôhoku Math. J. (2) 9 (1957), 119-221.

[7] Noriaki Kawanaka, "A theorem on finite Chevalley groups", Osaka $J$. Math. 10 (1973), 1-13.

[8] George Lusztig, "Unipotent characters of the symplectic and odd orthogonal groups over a finite field", Invent. Math. 64 (1981), 263-296. 
[9] George Lusztig, "Unipotent characters of the even orthogonal groups over a finite field", Trans. Amer. Math. Soc. 272 (1982), 733-751.

[10] George Lusztig, Representations of finite Chevalley groups

(Conference Board of the Mathematical Sciences Regional

Conference Series in Mathematics, 39. American Mathematical Society, Providence, Rhode Island, 1978).

[11] Louis Solomon, "A decomposition of the group algebra of a finite Coxeter group", J. Algebra 9 (1968), 220-239.

[12] Louis Solomon, "The Steinberg character of a finite group with $B N-$ pair", Theory of finite groups, 213-221 (Proc. Sympos., Harvard University, 1968. Benjamin, New York, 1969).

[13] T.A. Springer and R. Steinberg, "Conjugacy classes", Seminar on algebraic groups and related finite groups, Princeton, 1968/69, 167-226 (Lecture Notes in Mathematics, 131. Springer-Verlag, Berlin, Heidelberg, New York, 1970).

[14] Richard P. Stanley, "Some aspects of groups acting on finite posets", J. Combin. Theory Ser. A 32 (1982), 132-161.

[15] David B. Surowski, "Permutation characters of finite groups of Lie type", J. Austral. Math. Soc. Ser. A 27 (1979), 378-384.

[16] David B. Surowski, "A reflection sieve for finite $B N$-pairs of type $A_{n}, B_{n}$ and $C_{n} "$, J. Algebra 73 (1981), 538-551.

[17] Jean-Louis Verdier, "Caractéristique d'Euler-Poincaré", Bulz. Soc. Math. Fronce 101 (1973), 441-445.

Department of Pure Mathematics, University of Sydney,

Sydney,

New South Wales 2006,

Australia. 